\title{
Estudio comparativo de biocompatibilidad entre la hemodiafiltración en línea y la hemodiafiltración con reinfusión endógena
}

José Luis Cobo Sánchez, Raquel Pelayo Alonso, Raquel Menezo Viadero, Ma Eugenia Cuadrado Mantecón, Camino Villa Llamazares, Magdalena Gándara Revuelta, Hortensia Cepa García, Araceli Saenz de Buruaga Perea, $\mathbf{M}^{\mathrm{a}}$ Elena Incera Setién, Rosa Ana Sainz Alonso, $\mathbf{M}^{\mathrm{a}}$ Yolanda Vicente Jiménez, $\mathbf{M}^{\mathrm{a}}$ Soraya Sánchez Cano, Mirian García Martínez, Rosa Alonso Nates, Blanca Amalia Paule Peñasco

Enfermeros/as. Servicio de Nefrología. Hospital Universitario Marqués de Valdecilla. Santander

\section{Resumen}

Objetivo: Comparar la biocompatibilidad entre la hemodiafiltración en línea (HDF) y la hemodiafiltración con reinfusión endógena (HFR).

Material y método: Estudio comparativo observacional en una población de 15 pacientes en hemodiálisis crónica elegidos al azar entre los pacientes de nuestra unidad. Se compararon cambios en el perfil hematológico, nivel de PCR y constantes vitales, pre y post hemodiálisis, tras someterse a ambas técnicas de hemodiafiltración. Se comparó las diferencias entre los parámetros estudiados pre y post hemodiálisis en cada técnica.

Resultados: Los niveles de plaquetas descendieron más en la HDF (HDF -1,33 vs HFR $-19,73 \times 10^{3} / \mathrm{mm}^{3}$, $p=0,005)$. El nivel de leucocitos disminuyó en la HDF y aumentó en la HFR (HDF - 0,46 vs HFR $+0,8 \times 10^{3}$ / $\mathrm{mm}^{3} ; \mathrm{p}=0,006$ ). Respecto a la fórmula leucocitaria hubo resultados dispares: segmentados HDF -1,7 vs HFR $+5,4 \%, p<0,001$; linfocitos HDF $+1,96$ vs HFR $-3,62 \%, p<0,001$. Con la HFR disminuyeron menos los niveles de PCR (HDF -0,05 vs HFR $-0,001 \mathrm{mg} /$ $\mathrm{dl} ; \mathrm{p}=\mathrm{NS}$ ). En lo referente a las constantes vitales,

\begin{tabular}{|c|}
\hline Correspondencia: \\
José Luis Cobo Sánchez \\
Servicio de Nefrología \\
Hospital Universitario Marqués de Valdecilla \\
Avenida Valdecilla s/n \\
39008. Santander. Cantabria \\
e-mail: jlcobosanchez@gmail.com
\end{tabular}

la tensión arterial sistólica descendió más en la HFR que en la HDF (HDF -9,93 vs HFR -10,33 mmHg; $p<0,001$ ), a la inversa que la diastólica (HDF $-5,2$ vs HFR $-3 \mathrm{mmHg} ; p=0,007$ ) y la frecuencia cardiaca (HDF $-1,46$ vs HFR $+1,73$ Ipm; $p=N S$ ). La temperatura corporal aumentó más con la HDF que con la HFR (HDF $+0,35$ vs HFR $+0,06{ }^{\circ} \mathrm{C} ; \mathrm{p}=\mathrm{NS}$ ).

Conclusiones: Según nuestros resultados la HFR parece más biocompatible que la HDF, probablemente derivado por la reinfusión exógena de la HDF.

\section{PALABRAS CLAVE: \\ - HEMODIAFILTRACIÓN EN LÍNEA \\ - HEMODIAFILTRACIÓN CON REINFUSIÓN \\ ENDÓGENA \\ - ENSAYO DE MATERIALES \\ - CAMBIOS EN LA TEMPERATURA CORPORAL \\ - RECUENTO DE LEUCOCITOS}

Biocompatibility comparative study between online hemodiafiltration and hemodiafiltration with endogenous reinfusion.

\section{Abstract}

Aim: To compare biocompatibility between online hemodiafiltration (HDF) and hemodiafiltration with endogenous reinfusion. 
Methods: Observational comparative study in a population of 15 chronic hemodialysis patients randomly selected among the patients in our unit. We compared changes in hematological profile, CRP level and vital signs, pre and post hemodialysis, after undergoing both hemodiafiltration techniques. Comparing the differences between the parameters studied before and after each hemodialysis technique.

Results: Platelet levels decreased more in the HDF (HDF $-1,33$ vs HFR $-19,73 \times 10^{3} / \mathrm{mm}^{3}, p=0,005$ ). Leukocyte levels decreased in the HDF and increased with HFR (HDF $-0,46$ vs HFR $+0,8 \times 10^{3} / \mathrm{mm}^{3}$; $p=0,006$ ). Regarding the leukocyte formula had mixed results: segmented HDF $-1,7$ vs HFR $+5,4 \%$, $p<0,001$; lymphocytes HDF $+1,96$ vs HFR $-3,62 \%$, $p<0,001$. With the HFR decreased CRP levels less (HDF $-0,05$ vs HFR $-0,001 \mathrm{mg} / \mathrm{dl} ; p=\mathrm{NS}$ ). Regarding vital signs, systolic blood pressure decreased more in the HFR than HDF (HDF $-9,93$ vs HFR $-10,33$ $\mathrm{mmHg} ; p<0,001)$, conversely that the diastolic blood pressure (HDF $-5,2$ vs HFR $-3 \mathrm{mmHg} ; p=0,007$ ), and heart rate (HDF $-1,46$ vs HFR $+1,73$ Ipm; $p=N S$ ). Body temperature increased more with the HDF that the HFR (HDF $+0,35$ vs HFR $+0,06{ }^{\circ} \mathrm{C} ; \mathrm{p}=\mathrm{NS}$ ).

Conclusions: According to our results the HFR seems more biocompatible than the HDF, probably derived by exogenous reinfusion of HDF.

\section{KEY WORDS \\ - ONLINE HEMODIAFILTRATION \\ - HEMODIAFILTRATON WITH ENDOGENOUS \\ REINFUSION \\ - MATERIALS TESTING \\ - BODY TEMPERATURE CHANGES \\ - LEUKOCYTE COUNT}

La hemodiafiltración en línea (HDF) es una técnica de diálisis que suma al transporte difusivo propio de una hemodiálisis estándar (HD) una cantidad significativa de transporte convectivo. Éste aporta un mayor aclaramiento de moléculas medias y grandes, difíciles de eliminar por difusión ${ }^{2}$. La retención de estas moléculas urémicas se ha asociado clásicamente a diversas complicaciones crónicas de los pacientes en hemodiálisis ${ }^{3-7}$. Varios estudios han demostrado la disminución de estas complicaciones al aplicar técnicas con mayor transporte convectivo $0^{4,5,8}$.

Las técnicas convectivas "on-line» utilizan una membrana biocompatible de alto flujo y un baño de diálisis ultrapuro. La permeabilidad de la membrana y la biocompatibilidad tienen una influencia determinante en la concentración plasmática de beta-2-microglobulina ${ }^{9,10}$, en el desencadenamiento de los fenómenos inflamatorios inducidos por la diálisis ${ }^{11}$ y en la aparición de complicaciones clínicas por amiloidosis del enfermo dializado ${ }^{12,13}$.

La HDF realiza un alto transporte convectivo reinfundiendo con líquido de diálisis y eliminando el ultrafiltrado; mientras que en la hemodiafiltración con reinfusión endógena (HFR) el líquido ultrafiltrado en la primera cámara es reinfundido tras ser regenerado en un cartucho que adsorbe toxinas urémicas en la segunda cámara donde se produce la difusión. La HFR incorpora una membrana en la primera cámara con un cut-off significativamente superior al de las membranas High Flux empleadas en la HDF convencional, eliminando la reinfusión exógena y enfrenta a la sangre del paciente a un nuevo componente como es la resina para la adsorción. Ambas son técnicas que se fundamentan en un alto transporte convectivo, pero en las que la sangre del paciente se ve sometida a procesos y materiales diferenciados. Por este motivo, nuestro objetivo fue comparar la biocompatibilidad entre la HDF y la HFR.

\section{Material y método}

\section{Introducción}

El contacto de la sangre con los componentes del circuito extracorpóreo de diálisis produce una respuesta inflamatoria organizada. Un producto es más biocompatible cuanto menor es la magnitud de estas reacciones adversas ${ }^{1}$.
Estudio comparativo observacional en una población de 15 pacientes en hemodiálisis crónica elegidos al azar entre los pacientes de nuestra unidad, que previamente se sometían a una técnica de alto transporte convectivo. Para evaluar las diferencias en cuanto a biocompatibilidad entre ambas técnicas, se compararon cambios en 
el perfil hematológico, nivel de PCR y constantes vitales (tensión arterial, frecuencia cardíaca y temperatura), pre y post hemodiálisis, tras someterse a una sesión con cada una de las técnicas a estudio: HDF y HFR. Para ello se midieron las variables a estudio, realizando extracciones sanguíneas pre y post HD que fueron analizadas en el mismo laboratorio centralizado de nuestro hospital.

La HDF se realizó en modo predilucional con una membrana de helixona de 1,8 $\mathrm{m}^{2}$, con reinfusión automática para que fuese lo más similar posible a la HFR. La HFR se realizó con el kit HFR Supra que consta de un dializador de polifenileno de $1,7 \mathrm{~m}^{2}$ (con una porosidad en la primera cámara de $35 \mathrm{KDa}$ ) y un cartucho adsorbente de resina estirénica $(40 \mathrm{ml}$ de resina en solución fisiológica con una superficie adsorbente de $700 \mathrm{~m}^{2} / \mathrm{ml}$ de resina), con reinfusión automática (en función de la presión transmembrana para evitar la hemoconcentración). Ambas sesiones se realizaron en las mismas condiciones en cuanto a dosis de heparina, acceso vascular y turno de diálisis en cada paciente. Se recogieron, además de los datos analíticos, datos sociodemográficos y referentes a las técnicas estudiadas. Se solicitó el consentimiento informado a los pacientes que participaron en el estudio.

Las variables a estudio fueron analizadas utilizando el paquete estadístico SPSS versión 15.0 para Windows. Se calcularon los valores medios de cada una de las variables a estudio pre y post HD y se compararon entre sí para cada una de las técnicas. Se comparó la media de las diferencias entre los parámetros estudiados pre y post hemodiálisis en cada técnica. Para la inferencia estadística se utilizó el test de la T de Student para muestras relacionadas. Para las variables categóricas se calcularon los porcentajes.

\section{Resultados}

De los 15 pacientes estudiados el $60 \%$ fueron hombres, con una edad media 66 años (desviación típica 17,27 años). El $47 \%$ era portador de fístula. El 53,3\% se sometía previamente a PHF, el $20 \%$ a HDF predilucional y el $20,3 \%$ a HDF postdilucional. En cuanto a la nefropatía, la más común fue la vascular (40\%), seguida de la glomerular $(26,7 \%)$.

Por las características de las técnicas, hubo diferencias significativas en cuanto al volumen de sustitución: HDF
28,33 litros vs HFR 11,54 litros; $p<0,001$. Durante la HFR se dializó un volumen de sangre significativamente mayor que durante la HDF (HDF 71,17 litros vs HFR 76,06 litros; $p=0,01$ ). En cuanto al volumen ultrafiltrado no hubo diferencias estadísticamente significativas entre ambas técnicas (HDF 1970 ml vs HFR $2162 \mathrm{ml}$; $p=0,397$ ).

En la tabla 1 se muestran los valores medios hematológicos y de PCR, pre y post HD junto con la significación estadística al comparar dichos valores, para cada tipo de técnica. En la tabla 2 se muestran los valores medios de la diferencia entre los valores hematológicos y de PCR pre y post HD (valores positivos indican una disminución del parámetro estudiado y valores negativos indican un aumento), junto con la significación estadística al comparar dichos valores. En la tabla 3 se muestran los valores medios de las constantes vitales, pre y post HD junto con la significación estadística al comparar dichos valores, para cada tipo de técnica. En la tabla 4 se muestran los valores medios de la diferencia entre los valores de las constantes vitales pre y post HD (valores positivos indican una disminución del parámetro estudiado y valores negativos indican un aumento), junto con la significación estadística al comparar dichos valores.

\begin{tabular}{|c|c|c|c|c|c|c|}
\hline Parámetro & $\begin{array}{l}\text { HDF } \\
\text { pre }\end{array}$ & $\begin{array}{l}\text { HDF } \\
\text { post }\end{array}$ & p & $\begin{array}{l}\text { HFR } \\
\text { pre }\end{array}$ & $\begin{array}{l}\text { HFR } \\
\text { post }\end{array}$ & $\mathbf{p}$ \\
\hline $\begin{array}{l}\text { Hemoglobina } \\
(\mathrm{mg} / \mathrm{dl})\end{array}$ & 11,58 & 12,1 & 0,012 & 11,6 & 12,3 & 0,006 \\
\hline $\begin{array}{l}\text { Hematocrito } \\
(\%)\end{array}$ & 34,9 & 36,5 & 0,006 & 35,15 & 37,4 & 0,006 \\
\hline $\begin{array}{l}\text { Plaquetas } \\
\left(x 10^{3} / \mathrm{mm}^{3}\right)\end{array}$ & 167 & 165,7 & 0,524 & 175,4 & 155,6 & 0,006 \\
\hline $\begin{array}{l}\text { Leucocitos } \\
\left(x 10^{3} / \mathrm{mm}^{3}\right)\end{array}$ & 6,52 & 6,06 & 0,082 & 6,7 & 7,5 & 0,07 \\
\hline $\begin{array}{l}\text { Segmentados } \\
(\%)\end{array}$ & 68,78 & 67,07 & 0,165 & 68,52 & 73,92 & 0,003 \\
\hline $\begin{array}{l}\text { Linfocitos } \\
(\%)\end{array}$ & 18,53 & 20,81 & 0,057 & 19,28 & 15,66 & 0,011 \\
\hline $\begin{array}{l}\text { Monocitos } \\
(\%)\end{array}$ & 8,09 & 7,95 & 0,644 & 8,34 & 6,84 & 0,002 \\
\hline $\begin{array}{l}\text { Esosinófilos } \\
(\%)\end{array}$ & 3,6 & 3,5 & 0,665 & 3,3 & 2,94 & 0,158 \\
\hline $\begin{array}{l}\text { Basófilos } \\
(\%)\end{array}$ & 0,67 & 0,64 & 0,648 & 0,54 & 0,49 & 0,389 \\
\hline $\begin{array}{l}P C R \\
(\mathrm{mg} / \mathrm{dl})\end{array}$ & 1,62 & 1,65 & 0,405 & 1,81 & 2,01 & 0,395 \\
\hline
\end{tabular}

Tabla 1. Valores hematológicos y de PCR pre y post hemodiálisis en cada tipo de técnica y su significación estadística (HDF: hemodiafiltración en línea, HFR: hemodiafiltración con reinfusión endógena) 


\begin{tabular}{|l|c|c|c|}
\hline Parámetro & HDF & HFR & p \\
\hline Hemoglobina (mg/dl) & $-0,51$ & $-0,72$ & 0,284 \\
\hline Hematocrito (\%) & $-1,58$ & $-2,26$ & 0,246 \\
\hline Plaquetas (x103/mm $)$ & 1,33 & 19,73 & 0,005 \\
\hline Leucocitos (x103/mm $)$ & 0,46 & $-0,8$ & 0,006 \\
\hline Segmentados (\%) & 1,7 & $-5,4$ & $<0,001$ \\
\hline Linfocitos (\%) & $-1,96$ & 3,62 & $<0,001$ \\
\hline Monocitos (\%) & 0,14 & 1,5 & 0,014 \\
\hline Esosinófilos (\%) & 0,087 & 0,36 & 0,273 \\
\hline Basófilos (\%) & 0,033 & 0,053 & 0,823 \\
\hline PCR (mg/dl) & 0,05 & 0,01 & 0,487 \\
\hline
\end{tabular}

Tabla 2. Diferencia media entre valores hematológicos y de PCR pre y post hemodiálisis para ambos tipos de técnica (HDF: hemodiafiltración en línea, HFR: hemodiafiltración con reinfusión endógena)

\begin{tabular}{|l|c|c|c|c|c|c|}
\hline Parámetro & $\begin{array}{c}\text { HDF } \\
\text { pre }\end{array}$ & $\begin{array}{c}\text { HDF } \\
\text { post }\end{array}$ & \multicolumn{1}{|c|}{$\mathbf{p}$} & $\begin{array}{c}\text { HFR } \\
\text { pre }\end{array}$ & $\begin{array}{c}\text { HFR } \\
\text { post }\end{array}$ & \multicolumn{1}{|c|}{ p } \\
\hline TAS $(\mathrm{mm} / \mathrm{Hg})$ & 128,3 & 118,4 & 0,16 & 132,2 & 121,8 & 0,17 \\
\hline TAD (mm/Hg) & 66,53 & 61,31 & 0,055 & 72,73 & 69,73 & 0,464 \\
\hline $\begin{array}{l}\text { Frecuencia } \\
\text { cardiaca (Ipm) }\end{array}$ & 74,66 & 73,2 & 0,64 & 74,2 & 75,9 & 0,404 \\
\hline $\begin{array}{l}\text { Temperatura } \\
\text { corporal }\left({ }^{\circ} \mathrm{C}\right)\end{array}$ & 35,7 & 36,06 & 0,005 & 35,8 & 35,8 & 0,624 \\
\hline
\end{tabular}

Tabla 3. Constantes vitales pre y post hemodiálisis en cada tipo de técnica y su significación estadística (HDF: hemodiafiltración en línea, HFR: hemodiafiltración con reinfusión endógena, TAS: tensión arterial sistólica, TAD: tensión arterial diastólica)

\begin{tabular}{|l|c|c|c|}
\hline Parámetro & HDF & HFR & p \\
\hline TAS $(\mathrm{mm} / \mathrm{Hg})$ & 9,93 & 10,33 & $<0,001$ \\
\hline TAD $(\mathrm{mm} / \mathrm{Hg})$ & 5,2 & 3 & 0,007 \\
\hline Frecuencia cardiaca $(\mathrm{Ipm})$ & 1,46 & $-1,73$ & 0,113 \\
\hline Temperatura corporal $\left({ }^{\circ} \mathrm{C}\right)$ & $-0,35$ & $-0,06$ & 0,765 \\
\hline
\end{tabular}

Tabla 4. Diferencia media entre constantes pre y post hemodiálisis para ambos tipos de técnica (HDF: hemodiafiltración en línea, HFR: hemodiafiltración con reinfusión endógena)

\section{Discusión}

Nuestros resultados muestran un aumento estadísticamente significativo de los valores de hemoglobina y hematocrito en ambas técnicas. Este aumento se debe a la hemoconcentración de la sangre, fenómeno que también ocurre durante la HD convencional. En ambas técnicas los niveles de plaquetas disminuyen, siendo esta disminución estadísticamente significativa en el caso de la HFR. Esto indica que ambas técnicas no activan la vía extrínseca de la coagulación ya que en ambos casos se utilizan fibras sintéticas de alta permeabilidad como membrana de diálisis, que provocan una menor activación plaquetaria que las membranas celulósicas.

Respecto al nivel de leucocitos, en la HDF disminuyeron mientras que en la HFR aumentaron. En cuanto a la fórmula leucocitaria hubo resultados dispares: los segmentados o neutrófilos disminuyeron con la HDF y aumentaron con la HFR, y los monocitos aumentaron con la HDF y disminuyeron con HFR, mientras que para el resto disminuyeron con ambas técnicas. Se ha observado que al inicio de la diálisis se produce una leucopenia transitoria debida a leucoagregación pulmonar, que se correlaciona temporalmente con la activación del complemento. El número de neutrófilos disminuye a los 2-5 minutos, alcanza un máximo a los 15-20 minutos y revierte a los 60 minutos, y al final de la diálisis se observa un rebote, que alcanza aproximadamente a un $\mathbf{1 3 0 \%}$ de los que había prediálisis ${ }^{1}$. Nuestros resultados muestran por lo tanto que con la HDF ese rebote es menor que con la HFR. EI grado de leucopenia, preferentemente neutropenia, está íntimamente relacionado con el tipo de membrana. Cuando los pacientes se dializan con membranas celulósicas modificadas y polímeros sintéticos la leucopenia es menor que con el cuprofán, y es mínima con las membranas sintéticas de alta permeabilidad ${ }^{1}$. Nuestros resultados por lo tanto indican que con la HFR se produce una menos leucopenia que con la HDF.

Las cifras de tensión arterial sistólica disminuyen en ambas técnicas, pero en mayor medida en la HFR. Así mismo, la tensión arterial diastólica disminuye con ambas técnicas, de manera más marcada en el caso de la HDF. En cuanto a la frecuencia cardiaca, con la HFR aumentaba mientras que con la HDF disminuía, sin llegar a ser estadísticamente significativo. Esto podría estar relacionado con la ultrafiltración, sin embargo, a pesar de que se ultrafiltró mayor volumen con la HFR, esta diferencia no fue estadísticamente significativa. En el estudio de Álvarez y cols ${ }^{14}$ sobre los resultados obtenidos con la HFR, observaron una disminución de las cifras de tensión arterial, sin llegar a tener repercusión clínica en los pacientes, $y$ tolerando mejor la HD aquellos pacientes que previamente presentaban inestabilidad hemodinámica. 
En ese mismo estudio la frecuencia cardiaca también aumentaba ligeramente. Aunque no fue una variable a estudio en este trabajo, tenemos la sensación subjetiva de que nuestros pacientes presentaron mejor tolerancia hemodinámica con la HFR que con la HDF.

La temperatura corporal de los pacientes aumentó con ambas técnicas, pero con la HDF aumentó de manera estadísticamente significativa. Esto puede deberse a que con la HFR la reinfusión es endógena, mientras que con la HDF es exógena. En nuestra unidad utilizamos agua ultrapura, con un doble proceso de ósmosis inversa y un estricto control microbiológico, sin embargo, con la HDF se está poniendo en contacto la sangre del paciente con líquido de diálisis mientras que con la HFR esto no ocurre, identificándose el líquido de reinfusión como propio. Ésta es una de las mayores ventajas que ofrece la HFR frente a la HDF.

En cuanto a los niveles de PCR, disminuyen con las dos técnicas. Si tenemos en cuenta que la PCR es un marcador inflamatorio, podemos decir que con ambas técnicas éste se elimina y no se produce respuesta inflamatoria a sus componentes en cuanto a este parámetro. Panichi y cols ya observaron que con ambas técnicas se eliminaban grandes cantidades de interleuquinas, citoquinas $y$ PCR a lo largo del tiempo; por lo que concluyeron que ambas técnicas son muy biocompatibles ${ }^{15}$. Otros estudios a largo plazo, han demostrado que la HFR disminuye los niveles de leptina, adenopectina y beta-2-microglobulina, que induce a un menor estrés oxidativo que la HD con polisulfona ${ }^{16-18}$.

No hemos encontrado estudios en la literatura que utilicen nuestra misma metodología para comparar ambas técnicas en cuanto a parámetros de biocompatibilidad pre y post HD, pero los estudios indicados anteriormente muestran resultados a favor de una mayor biocompatibilidad de la HFR.

El reducido número de pacientes de nuestra investigación puede suponer una limitación del mismo, pero esta circunstancia se da también en los estudios consultados sobre los efectos de la HFR a medio plazo.

\section{Conclusiones}

Los resultados de nuestra investigación muestran un menor rebote del número de neutrófilos, una menor leucopenia, y un aumento menor de la temperatura corporal con la HFR que con la HDF. Por lo tanto, según nuestros resultados, la HFR parece más biocompatible que la HDF, probablemente derivado por la reinfusión exógena de la HDF.

Recibido: 20 Septiembre 2012

Revisado: 24 0ctubre 2012

Modificado: 10 Noviembre 2012

Aceptado: 12 Noviembre 2012

\section{Bibliografía}

1. Martín Malo A, de Francisco ALM. Dializadores y membranas de diálisis. En: Lorenzo V, LópezGómez JM, de Francisco ALM, Hernández D. Nefrología al día. Madrid: Grupo Editorial Nefrología de la Sociedad Española de Nefrología; 2010. p. 425-435.

2. López Gómez JM, Jofré R. Hemodiafiltración en línea. Tratado de hemodiálisis. Segunda edición actualizada. 2006; 13: 287-302.

3. Penne EL, Bankestijn PJ, Bots ML, the CONTRAST study group. Effect of increased convective clearance by on-line hemodiafiltration on all cause and cardiovascular mortality in chronic haemodialysis patients- the Dutch CONvective TRAnsport Study (CONTRAST): rationale and design of a randomized controlled trail [ISRNCTN38365125]. Curr Control Trials Cardiovasc Med 2005;20(6):8.

4. Vaslaski L, Major L, Berta K. On line haemodiafiltration versus haemodialysis; stable haematocrit with less erythropoietin and improvement of other relevant blood parameters. Blood Purif 2006;24:163-73.

5. Altieri $P$, Sorba G, Bolasco G, Asproni E, Ledebo I, Boström $M$, et al. Predilution hemofiltrationThe second Sardinian Study: comparisons between hemofiltration and haemodialysis during identical $\mathrm{Kt} / \mathrm{V}$ and sessions times in a long-term crossover 
study. Nephrol Dial Transplant 2001;16:1207-13.

6. Bonforte G, Grillo P, Zerbi S, Surian M. Improvement of anemia in hemodialysis patients treated by hemodiafiltration with high volumen on-line-prepared substitution fluid. Blood Purif 2002;20:35763.

7. Blankestijn PJ, Ledebo I, Canaud B. Hemodiafiltration: clinical evidence and remaining questions. Kidney Int 2010;77:581-7.

8. Lornoy W, Becaus J, Billiouw M, Sierens L, Van Malderen $P_{1}, D^{\prime}$ Haenens P. On-line haemodiafiltration. Remarkable removal of B2-microglobulin. Long-term clinical observations. Nephrol Dial Transplant 2000; 15 (Supl. 1): 49-54.

9. Grupo de Trabajo del Estudio Multicéntrico Español: Estudio Multicéntrico Español en diálisis corta con membrana de AN 69. Nefrología 1990;10(Supl 3):100-13.

10. Locatelli F, Andrulli S, Pecchini F, Pedrini L, Agliata $S$, Lucchi $L$, et al. Effect of high-flux dialysis on the anaemia of haemodialysis patients. Nephrol Dial Transplant 2000;15:1399-1409.

11. Ismail N. Biochemical mechanisms involved in blood-hemodialysis membrane interactions. UpToDate 2011. www.uptodate.com

12. Schiff $H$, Fischer $R$, Lang $S M$, Mangel E. Clinical manifestations of $A B$-amyloidosis: Effects of biocompatibility and flux. Nephrol Dial Transplant 2000;15:840-5.
13. Nakai S, Iseki K, Tabei K, Kubo K, Masakane I, Fushimi $\mathrm{K}$, et al. Outcomes of hemodiafiltration based on Japanese dialysis patient registry. Am J Kidney Dis 2001;38(Suppl 1): S212-S216.

14. Álvarez Serrano MD, Ludeña García MJ, Arnau Vives MJ. Nuevas terapias en hemodiálisis: HFR aequilibrium. Rev Soc Esp Enferm Nefrol 2010; 13 (4): 247-251.

15. Panichi V, Manca-Rizza G, Paoletti S, Taccola D, Consani $C$, Filippi $C$ et al. Effects on inflammatory and nutritional markers of haemodiafiltration with online regeneration of ultrafiltrate (HFR) vs online haemodiafiltration: a cross-over randomized multicentre trial. Nephrol Dial Transplant. 2006; 21(3):756-62.

16. Kim S, Oh KH, Chin HJ, Na KY, Kim YS, Chae DW, Ahn $C$ et al. Effective removal of leptin via hemodiafiltration with on-line endogenous reinfusion therapy. Clin Nephrol. 2009; 72(6):442-8.

17. González-Diez B, Cavia M, Torres G, Abaigar P, Muñiz $P$. Effect of a hemodiafiltration session with on-line regeneration of the ultrafiltrate on oxidative stress. Comparative study with conventional hemodialysis with polysulfone. Blood Purif. 2008;26(6):505-10.

18. Calò LA, Naso A, Carraro G, Wratten ML, Pagnin $E$, Bertipaglia $L$ et al. Effect of haemodiafiltration with online regeneration of ultrafiltrate on oxidative stress in dialysis patients. Nephrol Dial Transplant. 2007; 22(5):1413-9. 